\title{
Survival outcomes in prostate cancer patients with a prior cancer
}

\author{
Yan Zang ${ }^{1 \#}$, Feng $\mathrm{Qi}^{2 \#}$, Yifei Cheng ${ }^{3 \#}$, Tian $\mathrm{Xia}^{4}$, Rongrong Xiao ${ }^{4},{\mathrm{Xiao} \mathrm{Li}^{2} \text {, Ningli Yang }}^{1}$ \\ ${ }^{1}$ Department of Bariatric and Metabolic Surgery, The First Affiliated Hospital of Nanjing Medical University, Nanjing, China; ${ }^{2}$ Department of \\ Urologic Surgery, Jiangsu Cancer Hospital \& Jiangsu Institute of Cancer Research \& Affiliated Cancer Hospital of Nanjing Medical University, \\ Nanjing, China; ${ }^{3}$ Department of Urology, The First Affiliated Hospital of Nanjing Medical University, Nanjing, China; ${ }^{4}$ Department of Oncology, \\ Jiangsu Cancer Hospital \& Jiangsu Institute of Cancer Research \& Affiliated Cancer Hospital of Nanjing Medical University, Nanjing, China \\ Contributions: (I) Conception and design: X Li, N Yang; (II) Administrative support: None; (III) Provision of study materials or patients: Y Zang, X \\ Li, T Xia; (IV) Collection and assembly of data: F Qi, Y Zang, X Li; (V) Data analysis and interpretation: F Qi, Y Cheng, R Xiao; (VI) Manuscript \\ writing: All authors; (VII) Final approval of manuscript: All authors. \\ \#These authors contributed equally to this work. \\ Correspondence to: Xiao Li. Department of Urologic Surgery, Jiangsu Cancer Hospital \& Jiangsu Institute of Cancer Research \& Affiliated Cancer \\ Hospital of Nanjing Medical University, Nanjing 210009, China. Email: leex91@163.com; Ningli Yang. Department of Bariatric and Metabolic \\ Surgery, The First Affiliated Hospital of Nanjing Medical University, Nanjing 210029, China. Email: njyangningli2002@163.com.
}

Background: To shed light on the survival outcomes of prostate cancer (PCa) patients diagnosed after a prior cancer and identify prognostic factors for overall survival (OS) and cancer-specific survival (CSS) in PCa patients.

Methods: In the primary group, a total of 1,778 PCa patients with a prior cancer were identified in the Surveillance, Epidemiology, and End Results (SEER) database from 2005 to 2015, retrospectively. Baseline characteristics and causes of death (COD) of these patients were collected and compared. In the second group, a total of 10,296 PCa patients [5,148 patients with $\mathrm{PCa}$ as the only malignancy and 5,148 patients with PCa as their second primary malignancy (SPM)] diagnosed between 2010 and 2011 were extracted to investigate the impact of prior cancers on survival outcomes.

Results: In PCa patients with a prior cancer, the most common type of prior cancer was from gastrointestinal system (29.92\%), followed by urinary system (21.37\%). Patients were more likely to die of the prior caner, and those with prior cancer from respiratory system had the worst survival outcomes. Moreover, the overall ratios in patients with stage (PCa) I-II and III-IV diseases were 0.21 and 1.65, indicating that patients with higher stage diseases were more likely to die of PCa. In the second group, patients with PCa as the SPM had worse OS than those with PCa as the first primary cancer. Lastly, prognostic factors for OS and CSS in PCa patients were explored.

Conclusions: PCa remains to be an important COD for patients with a prior malignancy, especially for those with high-stage diseases. PCa patients with a prior cancer had worse survival outcomes than those without.

Keywords: Prostate cancer (PCa); Surveillance, Epidemiology, and End Results (SEER); survival; prior cancer; prognostic factor

Submitted Apr 27, 2020. Accepted for publication Dec 04, 2020.

doi: $10.21037 /$ tau-20-897

View this article at: http://dx.doi.org/10.21037/tau-20-897

\section{Introduction}

Prostate cancer ( $\mathrm{PCa}$ ) is one of the most common malignancies in genitourinary system globally (1). In
2020, the estimated newly diagnosed cases and deaths are 191,930 and 33,330 in the United States (2). In the United States and some European countries, the incidence rate of PCa has exceeded lung cancer to be the leading cause 
of male malignancies. Even though, disease progression of PCa could be well controlled by surgery, radiotherapy and endocrine therapy. It was reported that the 5-year overall survival (OS) rate for PCa was up to $90 \%$ in many institutions (3-5).

Overall, the 5-year relative survival rate of cancer survivors has been increasing during the past decades (up to $66 \%$ ) due to the improvement in cancer detection and treatment $(6,7)$. As a result, the number of cancer survivors is increasing recently. Statistically, the overall estimated cancer survivors in men and women were 7,377,100 and 8,156,120 in 2016 in the United States (8), and this population showed an annual growth trend of $2 \%$ (9). Considering the increasing number of cancer survivors, the probability of developing a second primary malignancy (SPM) also increased accordingly (10). Hence, many patients may develop tumors of multiple organs or systems during their lifetime (11).

An SPM is defined as a cancer which arises in a new organ or tissue independently at least 2 months after the initial diagnosis of the prior primary malignancy (12-14). Previous studies have already discussed the critical role of SPM in many cancer types, such as breast cancer $(11,15,16)$, Hodgkin lymphoma (17), cervical cancer (18) and so on. He et al. (19) found that there was an excessive risk of developing an SPM in young-onset (age $\leq 50$ years old) colorectal cancer survivors. Additionally, the risk of developing SPMs was reversely correlated to age. Donin et al. (20) demonstrated that about 1 in 12 patients would develop a second malignancy during their lifetime, and the most common type of SPMs was lung cancer. Moreover, they discovered that more than half of patients with two primary cancers died of the second malignancy totally.

Most previous studies have focused on the risk of developing an SPM after a known tumor. However, the risk of a specific tumor as an SPM in patients with a prior cancer and survival outcomes for these patients have not been widely discussed. Ji et al. (12) found that the most common type of prior cancer in breast cancer patients was gynecologic cancer, followed by gastrointestinal cancer. Besides, treatment for breast cancer significantly decreased the risk of breast cancer specific morality. As PCa was traditionally considered to be an indolent cancer, many cancer survivors or clinicians may not feel it worth treating after weighing the risks and benefits when it was diagnosed after another malignancy (21), and there were rare studies on this topic. Hence, we developed this study on the basis of the Surveillance, Epidemiology, and End Results (SEER) database to achieve a deeper understanding of the survival patterns and risk factors for patients with subsequent PCa. Additionally, we present the following article in accordance with the STROBE reporting checklist (available at http:// dx.doi.org/10.21037/tau-20-897).

\section{Methods}

All the raw data utilized in this study were retrospectively extracted from the SEER database. SEER registry is a public database supported by the US National Cancer Institute to collect relevant information of cancer patients, including demographic characteristics, incidence rates, treatments and survival outcomes. In the beginning, there were only nine regions participated in this project, while approximately $30 \%$ of the US population are covered in the database till now. In our study, we signed the user agreement and gained access to the database with the username of 15440-Nov2018. The study was conducted in accordance with the Declaration of Helsinki (as revised in 2013). Additionally, this study was exempt by Institutional Review Board (IRB) approval because the original data were from a public database and individual consent for this retrospective analysis was waived.

\section{Primary group}

In the primary group, $\mathrm{PCa}$ patients with a prior cancer were extracted from the SEER 9 registry using the "multiple primary-standard incidence ratio" function via the SEER*Stat software (Version 8.3.6; NCI, Bethesda, USA). The initial inclusion criteria were as follows: (I) PCa was the second malignancy of each patient, (II) patients with active follow-up after cancer diagnosis, (III) year of PCa diagnosis was from 2005 to 2015. Additionally, the exclusion criteria were as below: (I) patients with missing or unknown data [race $=13$, prostate-specific antigen $(\mathrm{PSA})=2,586$, Gleason score $=3,336$, stage $=2,334$, T stage $=255, \mathrm{~N}$ stage $=17, \mathrm{M}$ stage $=1$, cause of death $(\mathrm{COD})=1$ and the administration of surgery $=5$ ], (II) patients with three or more malignancies in total (n=3), (III) diagnosed by autopsy or death certificate only, (IV) diagnosis interval between PCa and the prior cancer was less than two months.

Afterwards, baseline characteristics and clinicopathological data were extracted for each patient, including age at diagnosis, race, histological type, marital status, types of the prior cancers, American Joint Committee on Cancer (AJCC) $6^{\text {th }}$ TNM stage, Gleason score, PSA level, diagnosis intervals between two cancers, administration of surgery, COD and 
follow-up. In this study, age at diagnosis was divided into $<65$ and $\geq 65$ years old. Race was classified into Black, White and Other (including American Indian/AK Native, Asian/ Pacific Islander). PSA was categorized into $\leq 4,4-10,10-20$ and $>20 \mathrm{ng} / \mathrm{mL}$. Gleason score fell into three categories: $\leq 6$, 7 and 8-10. Furthermore, prior cancers were classified based on different systems, such as gastrointestinal system, urinary system, respiratory system, oral cavity and so on. Finally, for patients who died during the follow-up, COD were categorized into $\mathrm{PCa}$, the prior cancer and other causes.

Firstly, the 5 most common types of the prior cancers were identified according to the frequency of occurrence, and Kaplan-Meier (KM) analyses were performed to probe the survival impacts of these cancers. Then, we calculated the percentage of PCa-related deaths and prior cancerrelated deaths in different cancer types. Furthermore, basic and pathological outcomes between patients who died of $\mathrm{PCa}$ and those died of the prior cancer were compared. Finally, the ratio of $\mathrm{PCa}$ deaths to prior cancer deaths was obtained for each prior cancer type, further stratified by PCa TNM stage.

\section{Second group}

In the second group, patients with histologically confirmed, stage I-III PCa from 2010 to 2011 were identified from the SEER 18 registry utilizing the "case listing session" tool. The enrolled patients were grouped into primary prostate cancer (PPC) and subsequent prostate cancer (SPC) according to whether there was a prior cancer before PCa diagnosis. The propensity score matching (PSM) method was developed with a ratio of 1:1 to balance the baseline characteristics. Comparisons between patients with PPC and SPC in survival outcomes were made to explore the impact of the prior cancers on survival. Finally, uni- and multivariate Cox regression analyses were constructed to identify the prognostic factors in PCa patients.

\section{Statistical analysis}

Student's $t$-test and chi-square analyses were used for the comparisons in baseline characteristics and clinicopathological data, respectively. Survival outcomes were compared utilizing the KM analyses. The whole analysis was performed via SPSS 23.0 software (SPSS Inc, Chicago, IL, USA) and R software (Version 3.4.1). A twosided $\mathrm{P}<0.05$ was considered to be statistically significant.

\section{Results}

\section{Baseline characteristics of the primary group}

A total of 1,778 eligible patients were included in the primary group. The median (interquartile range, IQR) ages at diagnosis of the prior caner and PCa were 64 [58-70] and 68 [63-74] years old. The median (IQR) diagnosis interval between two cancers was 40.5 [19-66] months. Overall, the majority of enrolled patients had their cancer diagnosed at earlier TNM stage (I-II: $76.94 \%$ and $86.33 \%$ for the prior cancer and $\mathrm{PCa}$, respectively). Besides, the median (IQR) follow-up after PCa diagnosis was 42 (23.00-63.75) months (Table 1). In the primary group, the 5 most common types of prior cancer were from gastrointestinal system (29.92\%), urinary system $(21.37 \%)$, skin $(19.97 \%)$, respiratory system (11.59\%) and oral cavity and pharynx (7.31\%) (Table 2). On the whole, a total of 299 patients died during the follow-up, and patients with prior cancer of respiratory system had the highest mortality (30.58\%).

\section{Survival outcomes in the primary group}

As shown in Figure 1, OS was significantly different in patients with different types of prior cancer $(\mathrm{P}<0.001)$. PCa patients with prior cancers of respiratory system had the worst survival outcomes [10-year OS: $59.1 \%$, $95 \%$ confidence interval (CI), 50.9-68.8\%], while those with prior cancers of skin owned the longest OS (10-year OS: 85.8\%, 95\% CI, 80.9-90.9\%).

On COD, 38.13\% of patients died of the prior cancer and $16.05 \%$ of patients died of PCa (Figure $2 A$ ). When stratified by cancer types, we found that in patients with cancers of respiratory system, the prior cancer-related death rate was the highest $(44.44 \%)$ and the PCa-related death rate was relatively lower $(12.70 \%)$. The highest $\mathrm{PCa}-$ related death rate $(19.64 \%)$ was found in patients with prior urological cancers. Hence, conclusions could be drawn that died of prior cancers was the main COD in these patients. Then, we compared the ratio of $\mathrm{PCa}$ deaths to prior cancer deaths in patients. As shown in Figure 2B, the overall ratios in patients with stage (PCa) I-II and III-IV diseases were 0.21 and 1.65 , indicating that patients with higher stage diseases were more likely to die of PCa. Analogously, similar trends were detected in the majority of cancer types. However, in patients with prior cancers of respiratory system, they may be more likely to die of the first primary malignancy regardless of the PCa TNM stage (the ratio was 0.22 and 0.60 in stage I-II and III-IV diseases, respectively). 
Table 1 Demographic and clinical factors of PCa patients with a prior cancer $(n=1,778)$

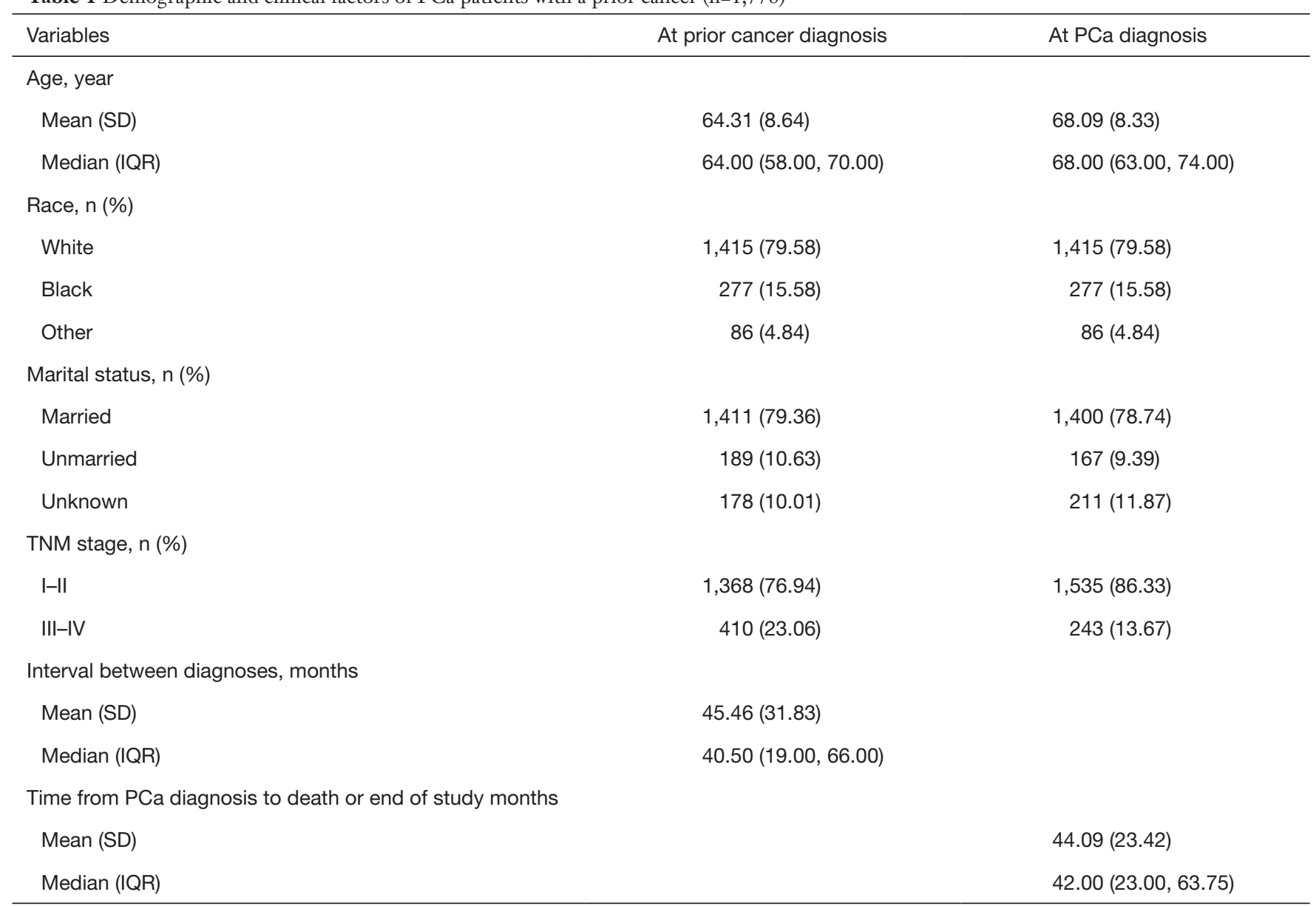

$\mathrm{IQR}$, interquartile range; $\mathrm{PCa}$, prostate cancer; SD, standard deviation.

Table 2 Classification of the prior malignancy, stratified by system

\begin{tabular}{llll}
\hline Systems & $\mathrm{N}(\%)$ & Detailed cancers & Death, $\mathrm{n}(\%)$ \\
\hline Gastrointestinal system & $532(29.92)$ & Esophagus, stomach, liver, colon, rectum and so on & $94(17.67)$ \\
Urinary system & $380(21.37)$ & Bladder, kidney, renal pelvic and ureter & $56(14.74)$ \\
Skin & $355(19.97)$ & Melanoma and other non-epithelial skin cancers & $39(10.99)$ \\
Respiratory system & $206(11.59)$ & Lung, bronchus, larynx and nose & 63 (30.58) \\
Oral cavity and pharynx & $130(7.31)$ & Tongue, tonsil, mouth and pharynx & $33(18.86)$ \\
Others & $175(9.84)$ & Others & 14 (8.00) \\
Overall & $1,778(100.00)$ & All of the above & 299 (16.82) \\
\hline
\end{tabular}

In Table 3, we found that age at $\mathrm{PCa}$ diagnosis $(\mathrm{P}<0.001)$, the rates of PSA $>20 \mathrm{ng} / \mathrm{mL}(\mathrm{P}<0.001)$, Gleason score $8-10$ $(\mathrm{P}<0.001)$, TNM stage III-IV $(\mathrm{PCa})$ diseases $(\mathrm{P}<0.001)$ and $\mathrm{Tx} / \mathrm{N} 1 / \mathrm{Mx}$ or $\mathrm{Tx} / \mathrm{Nx} / \mathrm{M} 1(\mathrm{PCa})$ diseases $(\mathrm{P}=0.026)$ were significantly higher in patients who died of PCa when compared with those who died of the prior cancer. Furthermore, the metastatic rate $(\mathrm{P}<0.001)$ of the prior cancer was significantly higher in patients who died of a prior cancer. 


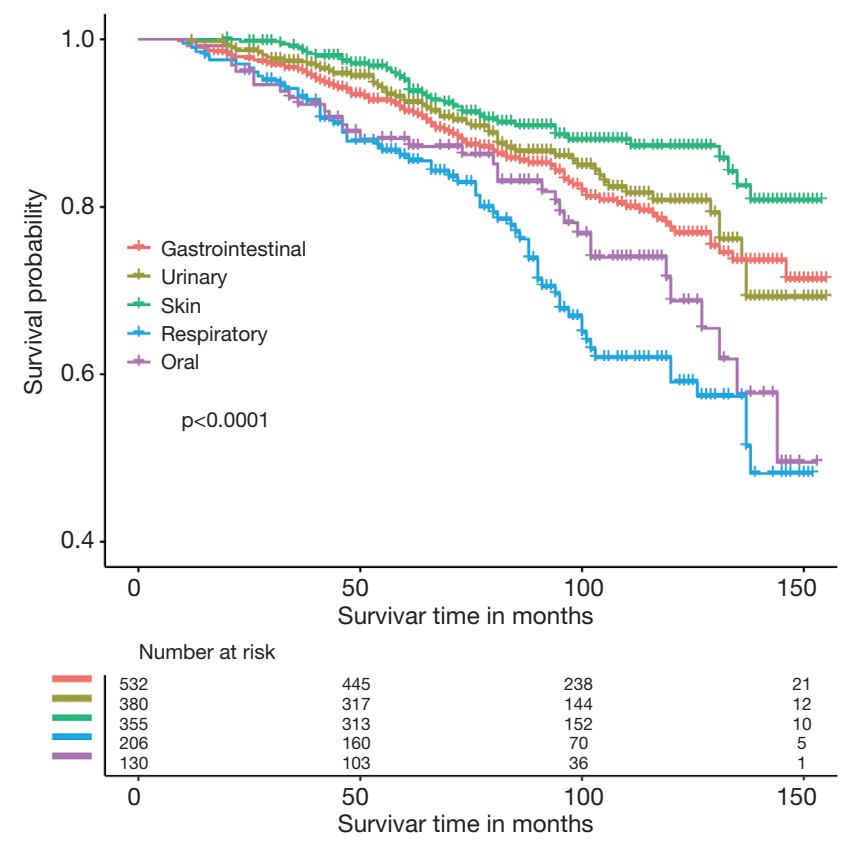

Figure 1 Overall survival of prostate cancer patients with a prior cancer.

\section{Survival of patients with PCa as the prior cancer or subsequent primary cancer in the second group}

A total of 72,173 patients were enrolled in the second group, including 67,025 patients had $\mathrm{PCa}$ as their first primary malignancy and 5,148 patients had PCa as the SPM. As shown in Table 4, significant differences were detected between two groups in many variables, including age at diagnosis, race, PSA level, Gleason score, TNM stage, marital status, administration of surgery and radiotherapy (all $\mathrm{P}<0.05$ ). To reduce the selection bias, a 1:1 PSM was developed and a total of 5,148 pairs of patients were eventually enrolled. As shown in Figure $3 A, B$, better survival outcomes were detected in patients with PPC when compared with those with SPC $(\mathrm{P}<0.05)$. After PSM, no significant difference was detected in prostate cancer-specific survival (PCSS) between two groups $(\mathrm{P}=0.66$, Figure $3 C$ ), while significant shorter OS was found in patients with SPC when compared with those with PPC $(\mathrm{P}<0.001$, Figure $3 D)$. Lastly, uni- and multivariate Cox regression analyses were conducted to explore prognostic factors associated to OS and PCSS in PCa patients. Multivariate analysis revealed that age at diagnosis, Gleason score, PSA level, TNM stage and administration of surgery were risk factors for cancer-specific survival (CSS) (all $\mathrm{P}<0.05$, Table 5). Similarly, age at diagnosis,
A

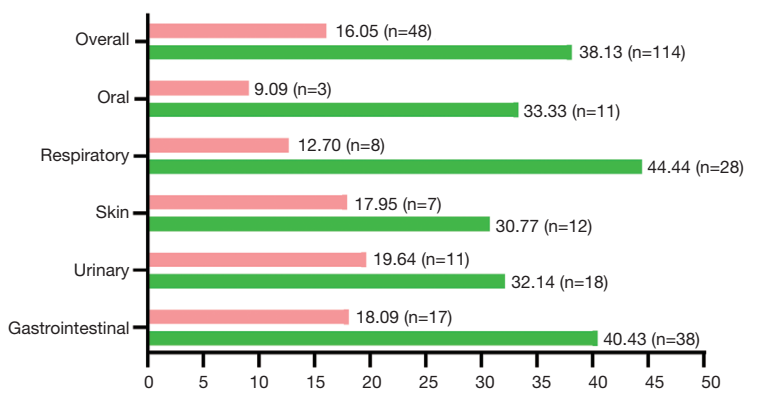

B

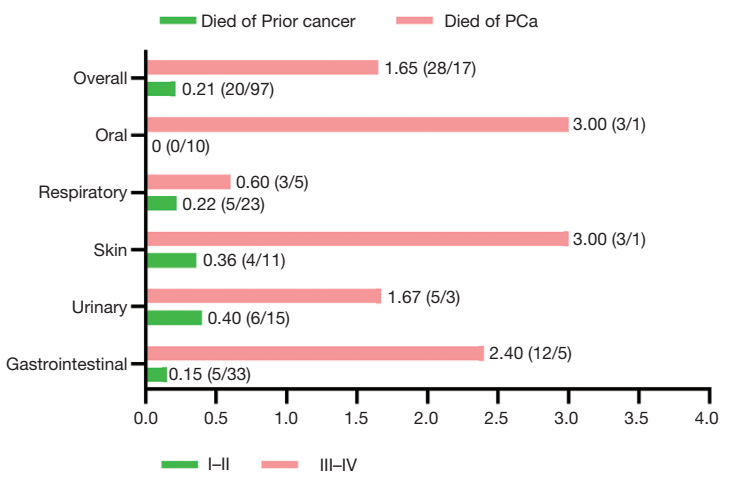

Figure 2 Survival outcomes among patients with different types of prior cancer. (A) The percentage of deaths related to prostate cancer or prior cancer among patients with different types of prior cancer, (B) ratio of prostate cancer deaths to prior cancer deaths.

race, Gleason score, PSA level, sequence of PCa (PPC vs. SPC) and administration of surgery were recognized as prognostic factors for $\mathrm{OS}$ (all $\mathrm{P}<0.05$, Table 6).

\section{Discussion}

Nowadays, with the increase of cancer survivors, the risk of developing SPMs has also been increasing accordingly. Additionally, prior cancer played an important role in treatment strategies and clinical trials design (22). It was traditionally accepted that patients with prior cancers should be excluded in clinical trials, which may due to the assumption that prior cancers may impact the survival outcomes $(23,24)$. Consequently, numerous patients with a prior cancer would be excluded from clinical trials, leading to worse accrual and generalizability of clinical trials (22). For example, up to about $20 \%$ of lung cancer patients were excluded from taking part in trails if following such a restrictive criterion (25). However, no convincing evidence has been proposed to support this exclusion criteria and address the actual effect of a prior malignancy on cancer survivors. Moreover, the standard incidence ratio of 
Table 3 Clinical and demographic factors associated with prostate cancer death $v$ s. prior cancer death

\begin{tabular}{|c|c|c|c|}
\hline Characteristics & Died from prior cancer & Died from PCa & $P$ value \\
\hline Age at $\mathrm{PCa}$ diagnosis, mean $\pm \mathrm{SD}$, year & $70.97 \pm 8.61$ & $77.29 \pm 9.07$ & $<0.001$ \\
\hline PCa treated, n (\%) & $22(19.30)$ & $13(27.08)$ & 0.272 \\
\hline Gleason score 8-10, n (\%) & $42(36.84)$ & $37(77.08)$ & $<0.001$ \\
\hline PCa, TNM stage III-IV, n (\%) & $17(14.91)$ & 28 (58.33) & $<0.001$ \\
\hline Prior cancer, TNM stage III-IV, n (\%) & $45(39.47)$ & $9(18.75)$ & 0.011 \\
\hline $\mathrm{PCa}, \mathrm{Tx} / \mathrm{N} 1 / \mathrm{Mx}$ or $\mathrm{Tx} / \mathrm{Nx} / \mathrm{M} 1, \mathrm{n}(\%)$ & $8(7.02)$ & $9(18.75)$ & 0.026 \\
\hline Prior cancer, Tx/N1-3/Mx or Tx/Nx/M1, n (\%) & $47(41.23)$ & $6(12.50)$ & $<0.001$ \\
\hline Gastrointestinal system & $38(33.33)$ & $17(35.41)$ & \\
\hline Urinary system & $18(15.79)$ & $11(22.92)$ & \\
\hline Skin & $12(10.53)$ & $7(14.58)$ & \\
\hline Respiratory system & $28(24.56)$ & $8(16.67)$ & \\
\hline Oral cavity and pharynx & $11(9.65)$ & $3(6.25)$ & \\
\hline Others & $7(6.14)$ & $2(4.17)$ & \\
\hline
\end{tabular}

PCa, prostate cancer; PSA, prostate-specific antigen; SD, standard deviation.

Table 4 Baseline characteristics of patients with PPC or SPC from the SEER database 2010-2011

\begin{tabular}{|c|c|c|c|c|c|c|}
\hline Variables & \multicolumn{3}{|c|}{ Data before PSM } & \multicolumn{3}{|c|}{ Data after PSM } \\
\hline$N$ & 67,025 & 5,148 & & 5,148 & 5,148 & \\
\hline Age (year) & & & $<0.001$ & & & 0.870 \\
\hline$<45$ & $427(0.64)$ & $18(0.35)$ & & $15(0.29)$ & $18(0.35)$ & \\
\hline$\geq 65$ & $34,237(51.08)$ & $3,548(68.92)$ & & $3,547(68.90)$ & 3,548 (68.92) & \\
\hline Race & & & $<0.001$ & & & 0.986 \\
\hline White & $52,811(78.79)$ & 4,413 (85.72) & & $4,414(85.74)$ & 4,413 (85.72) & \\
\hline Black & $10,761(16.06)$ & 554 (10.76) & & $556(10.80)$ & $554(10.76)$ & \\
\hline Grade I & $873(1.30)$ & $61(1.18)$ & & $59(1.15)$ & $61(1.18)$ & \\
\hline Grade II & $27,759(41.42)$ & $2,087(40.54)$ & & $2,082(40.44)$ & 2,087 (40.54) & \\
\hline Grade III & $38,300(57.14)$ & 2,994 (58.16) & & 2,999 (58.26) & 2,994 (58.16) & \\
\hline Grade IV & $93(0.14)$ & $6(0.12)$ & & $8(0.16)$ & $6(0.12)$ & \\
\hline
\end{tabular}

Table 4 (continued) 
Table 4 (continued)

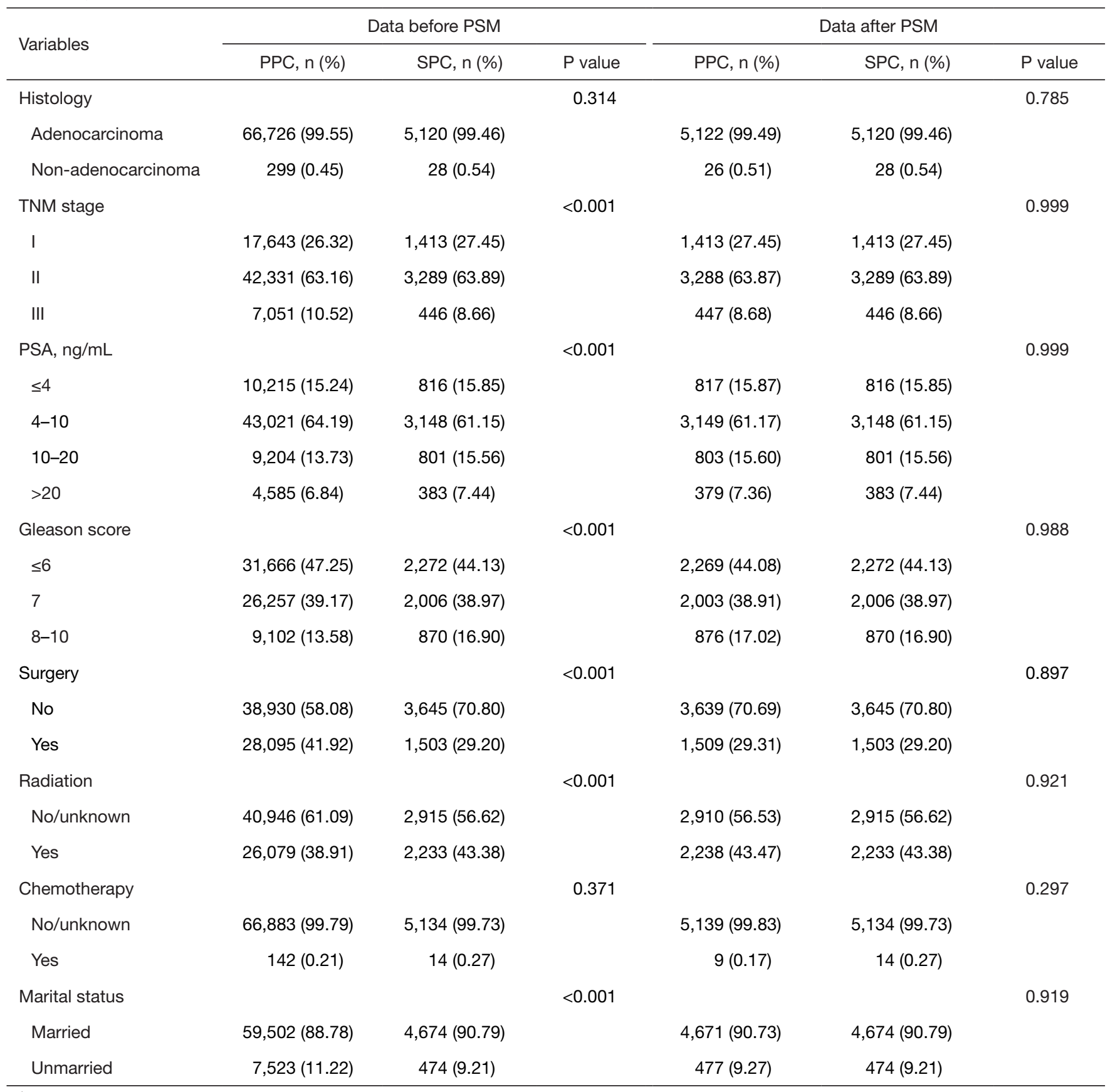

${ }^{1}$ Grade I, well differentiated; Grade II, moderately differentiated; Grade III, poorly differentiated; Grade IV, undifferentiated. SEER, Surveillance, Epidemiology, and End Results; PPC, primary prostate cancer; SPC, subsequent prostate cancer; PSA, prostate-specific antigen; PSM, propensity score matching; SD, standard deviation; SPC, subsequent primary cancer.

developing PCa after a prior cancer in the United States has been increasing in the past three decades (Figure S1). Considering that there are increasing cancer survivors develop PCa during the long-term follow up, it is necessary to investigate the survival outcomes of this population.

In this study, we found that the most common cancer type of prior cancers in PCa survivors was from gastrointestinal system. More patients died from their 

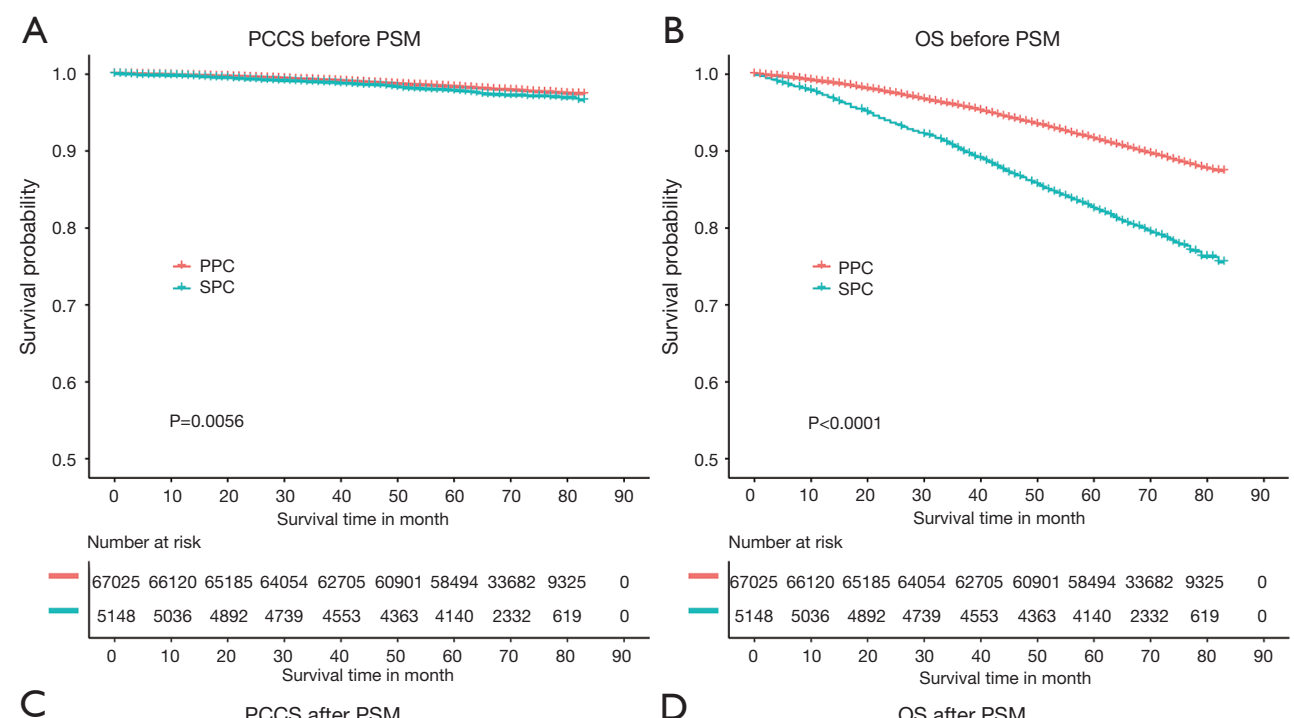

Number at risk
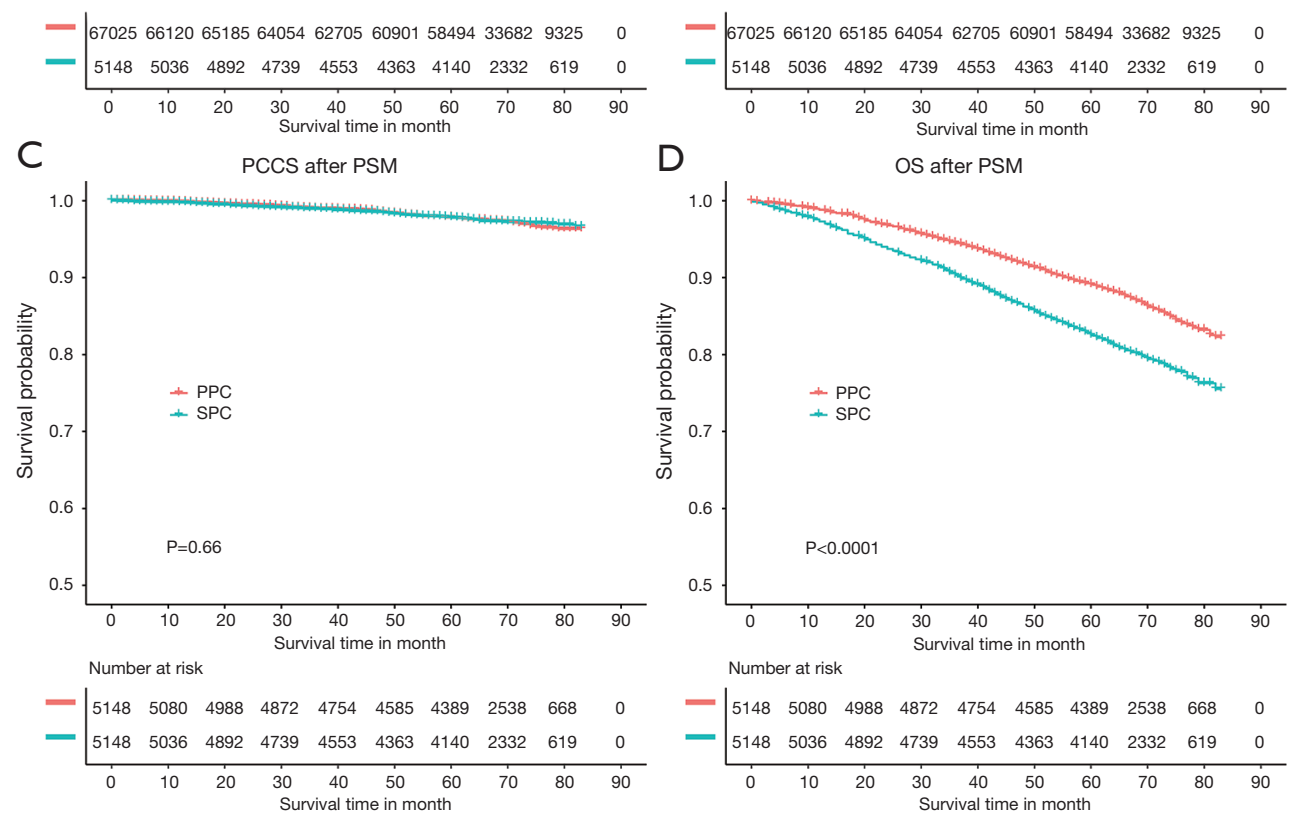

Figure 3 Kaplan-Meier survival curves of patients with prostate cancer as the second primary cancer or the prior cancer. (A,B) PCSS and overall survival before PSM; (C,D) PCSS and overall survival after PSM. PCSS, prostate cancer-specific survival; PSM, propensity score matching.

prior cancer rather than $\mathrm{PCa}(38.13 \%$ vs. $16.05 \%)$ with a median follow-up of 42 months, and this tendency existed in various systems. However, the ratio of PCa deaths to prior cancer deaths was greater than 1 in all systems except for respiratory system, suggesting that PCa remained to be an important COD in men with a prior caner, especially for those with stage III-IV PCa diseases. Nevertheless, in patients with a prior cancer of respiratory system, both patients and clinicians should focus on the treatment of the prior cancer rather than $\mathrm{PCa}$, regardless of the stage of PCa. Certainly, PCa patients with prior cancer of respiratory system had the shortest OS, while those with prior cancers of skin owned the longest OS. Similarly, Ji et al. (12) reported that breast cancer patients with prior cancers of lung had the worst OS, and those with prior melanoma had the best OS, with a median follow-up of 20.96 months. It was due to the fact that lung cancer was more lethal than many other cancers (26) and prior skin cancer in PCa patients could only affect the OS slightly. Laccetti et al. (24) demonstrated that the most common type of prior cancer in patients with locally advanced lung cancer was PCa (25\%), and prior PCa did not adversely affect OS in those patients. They claimed that locally advanced lung cancer patients with a prior cancer should not be excluded from clinical 
Table 5 Uni- and multivariate Cox regression model analysis of CSS

\begin{tabular}{|c|c|c|c|c|c|c|}
\hline Variables & \multicolumn{3}{|c|}{ Univariate } & \multicolumn{3}{|c|}{ Multivariate } \\
\hline Age, year & & & $<0.001$ & & & 0.012 \\
\hline$<65$ & Reference & & & Reference & & \\
\hline$\geq 65$ & 2.777 & $1.985-3.885$ & $<0.001$ & 1.562 & $1.104-2.211$ & 0.012 \\
\hline White & Reference & & & & & \\
\hline Black & 0.598 & $0.371-0.965$ & 0.035 & & & \\
\hline Other & 0.612 & $0.272-1.376$ & 0.235 & & & \\
\hline Grade $^{1}$ & & & $<0.001$ & & & 0.226 \\
\hline Marital status & & & 0.257 & & & \\
\hline Married & Reference & & & & & \\
\hline Unmarried & 0.764 & $0.479-1.217$ & 0.257 & & & \\
\hline Gleason score & & & $<0.001$ & & & $<0.001$ \\
\hline$\leq 6$ & Reference & & & Reference & & \\
\hline 7 & 1.956 & $1.351-2.830$ & $<0.001$ & 1.018 & $0.492-2.105$ & 0.962 \\
\hline $8-10$ & 9.800 & $7.025-13.673$ & $<0.001$ & 3.363 & $1.600-7.063$ & 0.001 \\
\hline $\mathrm{PSA}, \mathrm{ng} / \mathrm{mL}$ & & & $<0.001$ & & & $<0.001$ \\
\hline TNM stage & & & $<0.001$ & & & 0.070 \\
\hline I & Reference & & & Reference & & \\
\hline ॥ & 4.137 & $2.690-6.363$ & $<0.001$ & 1.300 & $0.690-2.452$ & 0.417 \\
\hline III & 5.245 & $3.125-8.804$ & $<0.001$ & 1.986 & $0.936-4.211$ & 0.074 \\
\hline Histology & & & 0.151 & & & \\
\hline Adenocarcinoma & Reference & & & & & \\
\hline Non-adenocarcinoma & 2.300 & $0.737-7.176$ & 0.151 & & & \\
\hline Diagnosis & & & 0.660 & & & \\
\hline PPC & Reference & & & & & \\
\hline SPC & 0.948 & $0.747-1.203$ & 0.660 & & & \\
\hline
\end{tabular}

Table 5 (continued) 
Table 5 (continued)

\begin{tabular}{|c|c|c|c|c|c|c|}
\hline Variables & \multicolumn{3}{|c|}{ Univariate } & \multicolumn{3}{|c|}{ Multivariate } \\
\hline Surgery & & & $<0.001$ & & & $<0.001$ \\
\hline No & Reference & & & Reference & & \\
\hline Yes & 0.297 & $0.205-0.430$ & $<0.001$ & 0.366 & $0.239-0.561$ & $<0.001$ \\
\hline
\end{tabular}

${ }^{1}$ Grade I, well differentiated; Grade II, moderately differentiated; Grade III, poorly differentiated; Grade IV, undifferentiated. CI, confidence interval; CSS, cancer-specific survival; HR, hazard ratio; PPC, primary prostate cancer; PSA, prostate-specific antigen; PSM, propensity score matching; SD, standard deviation; SPC, subsequent primary cancer.

Table 6 Uni- and multivariate Cox regression model analysis of OS

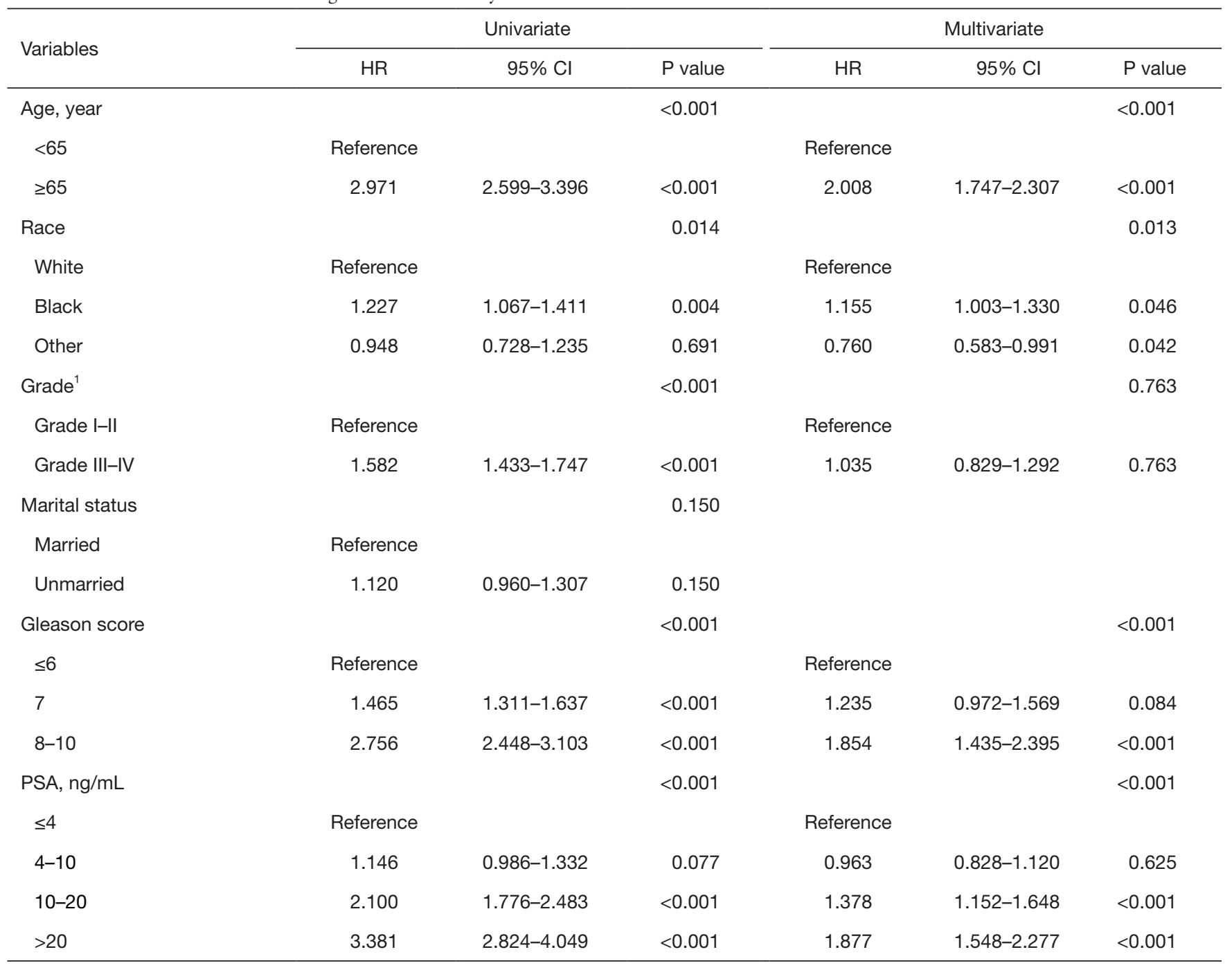

Table 6 (continued) 
Table 6 (continued)

\begin{tabular}{|c|c|c|c|c|c|c|}
\hline Variables & \multicolumn{3}{|c|}{ Univariate } & \multicolumn{3}{|c|}{ Multivariate } \\
\hline TNM stage & & & $<0.001$ & & & 0.299 \\
\hline I & Reference & & & Reference & & \\
\hline II & 1.641 & $1.461-1.843$ & $<0.001$ & 1.143 & $0.939-1.390$ & 0.183 \\
\hline Histology & & & 0.077 & & & \\
\hline Adenocarcinoma & Reference & & & & & \\
\hline Non-adenocarcinoma & 1.606 & $0.949-2.717$ & 0.077 & & & \\
\hline Diagnosis & & & $<0.001$ & & & $<0.001$ \\
\hline Surgery & & & $<0.001$ & & & $<0.001$ \\
\hline No & Reference & & & Reference & & \\
\hline Yes & 0.263 & $0.226-0.305$ & $<0.001$ & 0.347 & $0.292-0.412$ & $<0.001$ \\
\hline
\end{tabular}

${ }^{1}$ Grade I, well differentiated; Grade II, moderately differentiated; Grade III, poorly differentiated; Grade IV, undifferentiated. CI, confidence interval; OS, overall survival; HR, hazard ratio; PPC, primary prostate cancer; PSA, prostate-specific antigen; PSM, propensity score matching; SD, standard deviation; SPC, subsequent primary cancer.

trials, and they should be offered aggressive, potentially curative therapies if otherwise appropriate. We supposed that lung cancer played a leading role in survival outcomes in patients with both PCa and lung cancer. In our study, we recommended that $\mathrm{PCa}$ patients with prior cancers must be carefully considered for clinical trials.

Our results showed that patients with SPC had shorter OS when compared with those with $\mathrm{PPC}(\mathrm{P}<0.0001)$, while no significant difference was detected in PCSM $(\mathrm{P}=0.66)$. Zhou et al. (22) found that patients having PCa as an SPM had inferior OS than those having $\mathrm{PCa}$ as the only malignancy. Moreover, similar survival outcomes were found in those with other malignancies, including thyroid, bladder, kidney and renal pelvic, eye and orbits, breast and so on. In the study conducted by Ji et al. (12) concluded that patients with subsequent breast cancer had worse OS and breast CSS than those with primary breast cancer. However, no obvious difference was found in the CSS despite the statistical significance. Interestingly, significant better OS was detected in patients with second primary colorectal cancer than those with initial primary colorectal cancer (27). Moreover, Liu et al. (28) found that younger patients with lung cancers with a prior caner had the same or not-inferior OS than those without a prior cancer $(\mathrm{P}<0.05)$. We believed that these survival differences were due to the differences between PCa and lung cancer or colorectal cancer itself. Additionally, in this study, multivariate Cox regression revealed that sequence of PCa (PPC vs. SPC) was an independent prognostic factor for OS, but not for CSS, which was consistent with the result in KM-analysis. Therefore, researchers should be familiar with the past medical history of each patient, and pay more attention to patients with a prior cancer in clinical decisions.

However, there were some potential limitations that could not be ignored. Firstly, some data were missing in SEER database which limited further comprehensive analysis of the research, such as comorbidities (obesity, diabetes), cycle of radiotherapy and chemotherapy, chemotherapy drugs and so on. Secondly, treatment types of the prior cancer may affect the survival and occurrence of SPM (29). Lastly, although a PSM method was used in this study, unavoidable selection bias still existed due to the retrospective design. Thus, prospective and large sample size studies are needed to validate our findings in the future.

In conclusion, $\mathrm{PCa}$ is still an important COD for patients with a prior cancer, especially for those with high-stage diseases. In PCa patients with a prior cancer, the OS will be 
affected by the prior cancer significantly, indicating that we should be more prudential in clinical decision-making.

\section{Acknowledgments}

We were very grateful to Ji et al. because their works (Risk of breast cancer-related death in women with a prior cancer) gave us the inspiration to develop this research.

Funding: This study was supported by the National Natural Science Foundation of China (No. 81702520), Medical Research Project of Jiangsu Provincial Health and Family Planning Commission (No. H2018052), Research Project of Jiangsu Cancer Hospital (No. ZN201602), and the young talents program of Jiangsu Cancer Hospital (No. 2017YQL-04).

\section{Footnote}

Reporting Checklist: The authors have completed the STROBE reporting checklist. Available at http://dx.doi. org/10.21037/tau-20-897

Conflicts of Interest: All authors have completed the ICMJE uniform disclosure form (available at http://dx.doi. org/10.21037/tau-20-897). Dr. XL serves as an unpaid Section Editor of Translational Andrology and Urology from Jan 2020-Dec 2021. The other authors have no conflicts of interest to declare.

Ethical Statement: The authors are accountable for all aspects of the work in ensuring that questions related to the accuracy or integrity of any part of the work are appropriately investigated and resolved. The study was conducted in accordance with the Declaration of Helsinki (as revised in 2013). This study was exempt by Institutional Review Board (IRB) approval because the original data were from a public database and individual consent for this retrospective analysis was waived.

Open Access Statement: This is an Open Access article distributed in accordance with the Creative Commons Attribution-NonCommercial-NoDerivs 4.0 International License (CC BY-NC-ND 4.0), which permits the noncommercial replication and distribution of the article with the strict proviso that no changes or edits are made and the original work is properly cited (including links to both the formal publication through the relevant DOI and the license). See: https://creativecommons.org/licenses/by-nc-nd/4.0/.

\section{References}

1. Bray F, Ferlay J, Soerjomataram I, et al. Global cancer statistics 2018: GLOBOCAN estimates of incidence and mortality worldwide for 36 cancers in 185 countries. CA Cancer J Clin 2018;68:394-424.

2. Siegel RL, Miller KD, Jemal A. Cancer statistics, 2020. CA Cancer J Clin 2020;70:7-30.

3. Steele CB, Li J, Huang B, et al. Prostate cancer survival in the United States by race and stage (2001-2009): Findings from the CONCORD-2 study. Cancer 2017;123 Suppl 24:5160-77.

4. Guillaumier S, Peters M, Arya M, et al. A Multicentre Study of 5-year Outcomes Following Focal Therapy in Treating Clinically Significant Nonmetastatic Prostate Cancer. Eur Urol 2018;74:422-9.

5. Winter A, Sirri E, Jansen L, et al. Comparison of prostate cancer survival in Germany and the USA: can differences be attributed to differences in stage distributions? BJU Int 2017;119:550-9.

6. Wood ME, Vogel V, Ng A, et al. Second malignant neoplasms: assessment and strategies for risk reduction. J Clin Oncol 2012;30:3734-45.

7. Henderson TO, Amsterdam A, Bhatia S, et al. Systematic review: surveillance for breast cancer in women treated with chest radiation for childhood, adolescent, or young adult cancer. Ann Intern Med 2010;152:444-55; W144-54.

8. Miller KD, Siegel RL, Lin CC, et al. Cancer treatment and survivorship statistics, 2016. CA Cancer J Clin 2016;66:271-89.

9. Smyth EC, Tarazona N, Peckitt C, et al. Exclusion of Gastrointestinal Cancer Patients With Prior Cancer From Clinical Trials: Is This Justified? Clin Colorectal Cancer 2016;15:e53-9.

10. Park SM, Yun YH, Kim YA, et al. Prediagnosis Body Mass Index and Risk of Secondary Primary Cancer in Male Cancer Survivors: A Large Cohort Study. J Clin Oncol 2016;34:4116-24.

11. Jung SY, Kim YA, Jo M, et al. Prediagnosis obesity and secondary primary cancer risk in female cancer survivors: A national cohort study. Cancer Med 2019;8:824-38.

12. Ji F, Yang CQ, Li XL, et al. Risk of breast cancer-related death in women with a prior cancer. Aging (Albany NY) 2020;12:5894-906.

13. Lains I, Bartosch C, Mondim V, et al. Second Primary Neoplasms in Patients With Uveal Melanoma: A SEER Database Analysis. Am J Ophthalmol 2016;165:54-64.

14. Budnik J, Doucette C, Milano MT, et al. Survival of 
Patients With Second Primary Hodgkin Lymphoma. Clin Lymphoma Myeloma Leuk 2020;20:316-23.e2.

15. Li Z, Wu Q, Song J, et al. Risk of Second Primary Female Genital Malignancies in Women with Breast Cancer: a SEER Analysis. Horm Cancer 2018;9:197-204.

16. Matsuo K, Mandelbaum RS, Machida H, et al. Decreasing secondary primary uterine cancer after breast cancer: A population-based analysis. Gynecol Oncol 2019;154:169-76.

17. Kumar V, Garg M, Chandra AB, et al. Trends in the Risks of Secondary Cancers in Patients With Hodgkin Lymphoma. Clin Lymphoma Myeloma Leuk 2018;18:576-89.e1.

18. Rodriguez AM, Kuo YF, Goodwin JS. Risk of colorectal cancer among long-term cervical cancer survivors. Med Oncol 2014;31:943.

19. He $X, W u$ W, Ding $Y$, et al. Excessive risk of second primary cancers in young-onset colorectal cancer survivors. Cancer Med 2018;7:1201-10.

20. Donin N, Filson C, Drakaki A, et al. Risk of second primary malignancies among cancer survivors in the United States, 1992 through 2008. Cancer 2016;122:3075-86.

21. Lee DJ, Mallin K, Graves AJ, et al. Recent Changes in Prostate Cancer Screening Practices and Epidemiology. J Urol 2017;198:1230-40.

22. Zhou H, Huang Y, Qiu Z, et al. Impact of prior cancer history on the overall survival of patients newly diagnosed

Cite this article as: Zang Y, Qi F, Cheng Y, Xia T, Xiao R, Li X, Yang N. Survival outcomes in prostate cancer patients with a prior cancer. Transl Androl Urol 2021;10(2):741-753. doi: 10.21037/tau-20-897 with cancer: A pan-cancer analysis of the SEER database. Int J Cancer 2018;143:1569-77.

23. Murphy CC, Gerber DE, Pruitt SL. Prevalence of Prior Cancer Among Persons Newly Diagnosed With Cancer: An Initial Report From the Surveillance, Epidemiology, and End Results Program. JAMA Oncol 2018;4:832-6.

24. Laccetti AL, Pruitt SL, Xuan L, et al. Prior cancer does not adversely affect survival in locally advanced lung cancer: A national SEER-medicare analysis. Lung Cancer 2016;98:106-13.

25. Gerber DE, Laccetti AL, Xuan L, et al. Impact of prior cancer on eligibility for lung cancer clinical trials. J Natl Cancer Inst 2014;106:dju302.

26. Wood K, Hensing T, Malik R, et al. Prognostic and Predictive Value in KRAS in Non-Small-Cell Lung Cancer: A Review. JAMA Oncol 2016;2:805-12.

27. Yang L, Xiong Z, Xie QK, et al. Second primary colorectal cancer after the initial primary colorectal cancer. BMC Cancer 2018;18:931.

28. Liu J, Zhou H, Zhang Y, et al. Impact of prior cancer history on the overall survival of younger patients with lung cancer. ESMO Open 2020;5:e000608.

29. Davis EJ, Beebe-Dimmer JL, Yee CL, et al. Risk of second primary tumors in men diagnosed with prostate cancer: a population-based cohort study. Cancer 2014;120:2735-41. 